Need for Constant Visual Observation

\title{
Preliminary Evaluation of a Measure for Reliable Assessment of Need for Constant Visual \\ Observation in Adults with Traumatic Brain Injury
}

\author{
Anne Moessner, APRN-MS, CRRN \\ Mayo Clinic Department of Physical Medicine \& Rehabilitation \\ 200 1st Street SW, Rochester, MN, 55905 \\ Tel: 507-255-5109 \\ moessner.anne@mayo.edu
}

James F. Malec, PhD, ABPP-Cn, Rp (Corresponding author)

Physical Medicine and Rehabilitation,

Indiana University School of Medicine

And Rehabilitation Hospital of Indiana

Indianapolis, IN 46254

Tel: $317-329-2352$

jmalec@,rhin.com

Scott Beveridge BSN, RN, CRRN

UPMC Mercy Hospital Rehabilitation Institute

1400 Locust Street, Unit 6E

Pittsburgh, Pa 15219

Tel: 412-232-4026

beveridges@UPMC.edu

Cara Camiolo Reddy, MD, MMM

UPMC Mercy Hospital Rehabilitation Institute

1400 Locust Street, D-G103

Pittsburgh PA 15219

Tel: 412-232-8918

camice@upmc.edu

Tracy Huffman BSN, RN, CRRN

The Ohio State University Wexner Medical Center

410 West 10th Avenue, Columbus, OH 43210

Tel: 614-293-8000

Tracy.huffman@,osumc.edu

Julia Marton, OT

Baylor Institute for Rehabilitation-Dallas Campus (Department of Rehabilitation)

909 N. Washington Ave.

Dallas, TX 75246

Tel: 214-820-9270

JMarton@,bir-rehab.com

This is the author's manuscript of the article published in final edited form as:

Moessner, A., Malec, J. F., Beveridge, S., Reddy, C. C., Huffman, T., Marton, J., \& Schmerzler, A. J. (2016). Preliminary evaluation of a measure for reliable assessment of need for constant visual observation in adults with traumatic brain injury. Brain Injury, 30(11), 1343-1349. https://doi.org/10.1080/02699052.2016.1193629 
Audrey J Schmerzler, DNP, RN, CRRN

1190 5th Ave, New York, NY 10029

NYP/Cornell-Weill Medical Center

New York, New York 10065

Tel: 212-746-1635

ajs9032@,nyp.org

Acknowledgement: The authors are grateful to the following individuals for their assistance in study recruitment and implementation at the various study sites: Mary Ferraro, PhD, OTR/L, Lisa Pinder RN, BSN, CRRN, Rachelle Rigous RN, BSN, CRRN (Drucker Brain Injury Center, MossRehab); Mary Gordon, RN, BSN, CRRN, Leanne Scroggins, MSN, CRRN (Mayo Clinic, Department of Nursing); Batya Geft, PT, Jason van de Ven OTR/L BOT, Debra Zeitlin MS CCC/SLP CBIS (Mount Sinai Hospital, Department of Rehabilitation Medicine); Lynne M Genter, MS,RN,CRRN, Michele Rinkes, RN, CRRN (Ohio State University, Wexner Medical Center); Mark Williams, RN (Rehabilitation Hospital of Indiana, Department of Neuropsychology).

The contents of this report were developed under a grants from the National Institute on Disability, Independent Living, and Rehabilitation Research (NIDILRR) for the Baylor Institute for Rehabilitation, Dallas, TX; Mayo Clinic, Rochester, MN; MossRehab, Philadelphia, PA; Mount Sinai Rehabilitation Center, New York, NY; University of Pittsburgh Medical Center, Pittsburgh, PA; Indiana University/Rehabilitation Hospital of Indiana TBI Model System (USDE-90DP0036-01-00); and the Ohio Regional TBI Model System at Ohio State University, Columbus, OH (USDE-90DP0040-01-00). NIDILRR is a Center within the Administration for Community Living (ACL), Department of Health and Human Services (HHS). The contents of this report do not necessarily represent the policy of NIDILRR, ACL, HHS, and you should not assume endorsement by the Federal Government.

At the time this study was conducted, Ms. Schmerzler was employed by The Mount Sinai Hospital Department of Rehabilitation Medicine.

The authors have no conflicts of interest to report.

Reprints will not be available for this manuscript. 
Need for Constant Visual Observation

\title{
Preliminary Evaluation of a Measure for Reliable Assessment of Need for Constant Visual Observation in Adults with Traumatic Brain Injury
}

\begin{abstract}
Primary objective: To develop and provide initial validation of a measure for accurately determining the need for Constant Visual Observation (CVO) in patients with traumatic brain injury (TBI) admitted to inpatient rehabilitation. Research design: Rating scale development and evaluation through Rasch analysis and assessment of concurrent validity. Methods and procedures: 134 individuals with moderate-severe TBI were studied in 7 inpatient brain rehabilitation units associated with the National Institute for Disability, Independent Living, and Rehabilitation Research (NIDILRR) TBI Model System. Participants were rated on the preliminary version of the CVO Needs Assessment scale (CVONA) and, by independent raters, on the Levels of Risk (LoR) and Supervision Rating Scale (SRS) at 4 time points during inpatient rehabilitation: admission, Days 2-3, Days 5-6, and Days 8-9. Outcomes and results: After pruning misfitting items, the CVONA showed satisfactory internal consistency (Person Reliability $=.85-.88$ ) across time points. With reference to the LoR and SRS, low false negative rates (sensitivity $>90 \%$ ) were associated with moderate to high false positive rates $(29 \%-56 \%)$. Conclusions: The CVONA may be a useful objective metric to complement clinical judgment regarding the need for $\mathrm{CVO}$; however, further prospective study is desirable to further assess its utility in identifying at-risk patients, reducing adverse events, and decreasing CVO costs.
\end{abstract}




\section{Preliminary Evaluation of a Measure for the Reliable Assessment of Need for Constant Visual Observation in Adults with Traumatic Brain Injury}

Patients with traumatic brain injury (TBI) admitted to inpatient rehabilitation units often present with severe cognitive, behavioral and physical deficits, i.e., confusion, impaired memory, impulsivity, agitation, lack of insight, and impaired balance. As a result, patient safety often is compromised. According to Beaulieu et al. ${ }^{1}$ acute recovery following moderate to severe TBI is marked by alterations in responsiveness to the environment. Referred to as post-traumatic amnesia or confusion, this stage is a hallmark of early recovery and frequently results in the need for skilled nursing care in a hospital or brain rehabilitation unit. Agitated patients may resist direct care, be disruptive on the unit, and pose a physical risk to themselves, family, and staff all of which affect ability to engage in therapy. ${ }^{2-6}$ Physically restraining agitated patients often worsens behavior. Restraint use has been associated with decreased cognitive and psychological well-being and can increase risk of serious injury ayet not positively impact fall rates. ${ }^{7,8}$ In contrast, employing a multi-component approach to patient management resulted in reduced restraint use along with a decline in fall rate. ${ }^{9}$

Agitated and impulsive patients are often assigned 1:1 nurse-to-patient monitoring to reduce risk. Votruba and colleagues ${ }^{10}$ describe the effects of impulsivity in patients with TBI and implications for rehabilitation and safety including increased risk of adverse outcomes, such as, accidents and subsequent injuries. Impulsivity impedes the rehabilitation process which in turn extends length of stay.. These authors further suggest that there is no substitute for clinician observation to assess the multidimensional construct of impulsivity. Bogner et al. ${ }^{11}$ found that the presence of agitation measured by the Agitated Behavior Scale (ABS) was associated with increased length of hospital stay and poorer cognitive and motor functioning at discharge. The 
ABS is a scale commonly referenced in studies on TBI patient safety. ${ }^{6,12,13}$ In a sample of 51 patients on a single rehabilitation unit, Amato and colleagues ${ }^{12}$ demonstrated the feasibility, reliability and clinical utility of the ABS in considering the need for CVO but did not report the sensitivity and specificity or other detailed psychometric analyses of the measure.

Patients in acute rehabilitation are one of the most at risk groups for falls which carry huge financial implications. ${ }^{14}$ Following least restrictive guidelines, most rehabilitation centers curtail use of physical restraints. As a result, use of coaches, sitters, patient care assistants, or nursing assistants to provide 1:1 care, also known as constant visual observation (CVO), has skyrocketed without established evidence of cost-effectiveness. ${ }^{15-17}$ Eastwood and Schechtman ${ }^{18}$ estimated the costs of $\mathrm{CVO}$ on their 36 bed rehabilitation unit to be $\$ 6,000$ for a typical 3 week length of stay, or $\$ 78,000$ per year for each patient monitored.

In a survey conducted in preparation for this study, 26 nurse and therapy leaders from 18 Traumatic Brain Injury Model Systems (TBIMS) and one additional acute rehabilitation center (average $120 \mathrm{TBI}$ admits/year; range 30-226) found no agreed upon best practices for documenting the need for $\mathrm{CVO}$ or other less restrictive safety interventionsand no standardized $\mathrm{CVO}$ weaning protocols. Among the majority of centers, CVO decisions were based on interdisciplinary team communication with $73 \%$ of centers reassessing every 24 hours or more often. One therapist described it as "a lot of verbal report, not much objective data". Documentation of possible need for CVO was most often per one or more of the following: narrative shift notes (64\%), a "homegrown tool" (43\%), and/or the ABS (36\%). The ABS was seen as insufficient because while it measured agitation, it fell short of measuring other factors that justified CVO and its related staffing challenges and budgetary costs. It was also described as insensitive towards capturing more subtle changes in patient status, like improved orientation 
and self-awareness, and often did not play a prominent role in the decision to initiate or stop CVO. Verbal behaviors, while disruptive, did not justify the cost of 1:1 nursing care. Similarly, repetitive behaviors, resistance to care, sudden mood changes, pulling at tubes, and short attention span did not predictably justify 1:1 care. There was consensus that specific patient behaviors outside those measured on the ABS greatly contributed to CVO decision making and, while there were reasons to measure and track agitation, as one survey respondent stated, "if every patient that was agitated was placed on CVO, we'd be broke". In regards to weaning from CVO, many centers generally followed the Riedel and Shaw 1997 recommendations ${ }^{19}$ to engage in distancing, observing, coaching, shadowing, and informing once confusion diminished. The survey confirmed the suspicion that in many cases, once patients with TBI were placed on CVO, they were not weaned in a regimented or timely manner and that fear played a role according to $87 \%$ of respondents.

Pilot work at one of the TBIMS centers, the University of Pittsburgh Medical Center (UPMC), resulted in a new assessment tool based on the ABS. The UPMC tool incorporated additional items to enhance interdisciplinary team communication on patient safety. The scale also showed promise in identifying which patients needed CVO, which were ready to be weaned, and which did not require CVO but would benefit from other customary safety measures, such as, frequent checks or chair and bed alarms.

The study described here built on the work initiated by UPMC and another TBIMS center, Ohio State University, where the ABS was developed Study aims are consistent with national health care priorities to improve patient safety. ${ }^{20}$ Ultimately this study also sought to provide a means to decrease variability in practice through development and dissemination of a standardized patient assessment protocol. And, to promote development of weaning protocols, 
lead to more efficient use of resources, save cost, and help maximize patient safety. The specific aim of this study was to establish and provide initial validation of a measure for accurately determining the need for CVO of patients with TBI admitted to inpatient rehabilitation. An internally consistent measure of the need for CVO was developed and confirmed using Rasch analysis; the concurrent validity of the measure was assessed in relation to subjective clinical judgment recorded using the Pittsburgh Levels of Risk scale (LoR) and the current level of supervision as measured by the Supervision Rating Scale (SRS).

\section{Method}

IRB approval for the conducts of this study was received at each participating center.

\section{Participants}

134 individuals with moderate-severe TBI admitted to one of 7 inpatient brain injury rehabilitation units in the U.S. were participants in this study. Currently, all study sites are National Institute on Disability, Independent Living, and Rehabilitation Research (NIDILRR) funded Traumatic Brain Injury Model System centers, 6 of the 7 were at the time of the study.

Consecutive cases meeting the following inclusion/exclusion criteria were enrolled in the study at each site. Inclusion criteria: (1) diagnosis of moderate-severe TBI as per meeting one of the following criteria: initial Glasgow Coma Scale $<13$ or post-traumatic amnesia duration $>24$ hours or the presence of injury-related abnormalities on neuroimaging or admitted to inpatient rehabilitation with clinical diagnosis of moderate-severe TBI; (2) admission to an acute inpatient rehabilitation unit at a participating center; (3) age 18 years of age or greater. Exclusion criteria: (1) patients in a minimally conscious state (Ranchos Los Amigos Scale score of III or less) on admission; (2) patients on heavily sedating medications for agitation; (3) prisoners. 
Need for Constant Visual Observation

The sample included 44 (33\%) women and 90 (67\%) men with an average age of 46.8 years (SD =21.05). All participants met TBI Model Systems criteria for moderate-severe TBI (inclusion criterion \#1). Length of post-traumatic amnesia was available in 87 cases with a mean of 23.95 days $(\mathrm{SD}=25.14$ dys). An initial CT scan was recorded in 130 cases; 94\% (122/130) were positive.

\section{Measures}

Constant Visual Observation Needs Assessment (CVONA) incorporates the original 14 items from the ABS. The $\mathrm{ABS}^{22}$ is a reliable instrument for measuring agitation of individuals with TBI as well as residents in long-term care facilities experiencing dementia. Through a series of conference calls among this study's co-investigators and building on pilot information from the UPMC and prior research ${ }^{12}$ on inpatient rehabilitation unit fall risk factors, an additional 15 items were added to the ABS by consensus for a total of 29 items in the preliminary CVONA scale. The CVONA scale captured not only obvious agitated behaviors, such as, restlessness, pulling at tubes, short attention span, explosive anger, and impulsivity, but other safety risks common to patients with TBI including impaired balance, incontinence, poor short term memory, inability to reliably use a call light or otherwise express needs, and lack of awareness of deficits. Each item on the CVONA was rated on a 4-point scale: 1=absent; $2=$ present to a slight degree (The behavior is present but does not prevent the conduct of other, contextually appropriate behavior; the individual may redirect spontaneously, or the continuation of the agitated/unsafe behavior does not disrupt appropriate behavior); $3=$ present to a moderate degree (The individual needs to be redirected from an agitated/unsafe behavior to an appropriate behavior, but benefits from such cueing); 4=present to an extreme degree (The individual is not able to engage in appropriate/safe behavior even when external cueing or redirection is 
provided). The scale included 11 items describing potentially unsafe physical activities, 10 describing cognitive/communication impairments, and 8 describing problematic behavioral/emotional features. The final version of the CVONA is available on-line as supplementary material.

As we stated in the introduction to this paper, there is no generally agreed upon or gold standard method or procedure for assessing CVO. For this reason, the validity of the CVONA could only be assessed in comparison to concurrent measures that also appear related to the need for CVO. We chose two measures to assess the concurrent validity of the CVONA: the University of Pittsburgh Levels of Risk rating that represents subjective clinical judgment of the need for CVO, and the Supervision Rating Scale which is a rating of the degree of supervision that is currently provided to a patient. Both measures are described in greater detail below.

Levels of Risk (LorR). The UPMC pilot tool identifies four levels of risk for patients with TBI on an acute inpatient rehabilitation unit. In broad terms, the levels range from $1=$ no safety risk, no need for CVO to $4=$ extreme safety risk, definite need for CVO. The LoR helps direct the need for CVO or other less restrictive safety interventions. A copy of the LoR is available on-line as supplementary material.

Supervision Rating Scale (SRS) ${ }^{21}$ measures the level of supervision that a patient/participant receives from caregivers. The SRS rates level of supervision on a 13-point ordinal scale that can optionally be grouped into five ranked categories (Independent, Overnight Supervision, Part-Time Supervision, Full-Time Indirect Supervision, and Full-Time Direct Supervision). Because all participants in the current project were rehabilitation inpatients, only the ratings for "Full-Time Indirect Supervision" (levels 8-9) and "Full-Time Direct Supervision" 
(levels 10-13) were used. Ratings are based on the level of supervision currently received, not on how much supervision a participant is judged or predicted to need.

\section{Procedures}

This was a prospective, multi-site observational study designed for rating scale development. Participating brain rehabilitation centers were: Baylor Institute for Rehabilitation, Dallas, TX; Mayo Clinic, Rochester, MN; MossRehab, Philadelphia, PA; Mount Sinai Rehabilitation Center, New York, NY; Ohio State University/Dodd Rehabilitation, Columbus, $\mathrm{OH}$; Rehabilitation Hospital of Indiana, Indianapolis, IN; and University of Pittsburgh Medical Center, Pittsburgh, PA.

Prior to commencement of participant recruitment at each site, staff nurse and independent (therapists or co-investigators) raters underwent training that included study overview of recruitment protocols, inclusion/exclusion criteria, timing of assessments, and orientation to and practice with study measurement tools (LoR and SRS rated by nurses; CVONA rated by therapists and co-investigators). Training emphasized that the study was not intended to affect current nursing practice or local standards of care for assessing patient safety status, determining frequency of safety assessments, related documentation practices, weaning protocols, or assigning related interventions based on assessed need. Study investigators at each site were asked to identify a consecutive series of 20 adults with TBI who met study inclusion criteria. Only new admissions were considered.

Trained staff nurses (5-6) from each participating center assigned both the LoR and the SRS score for identified participants upon admission to the inpatient rehabilitation unit. Clinical judgment, review of the medical record, and discussion with staff from the sending hospital unit were used for assigning these scores. Admission scores were documented twice, on both the day and evening or night shift, to capture variability and because nursing staff interact with patients 
24 hours per day. Both of these admission scores were completed within 24 hours of the patient entering the rehabilitation unit.

An independent rater from a pool of 5-6 therapists and local co-investigators administered the proposed CVONA for the same identified participants. This initial rating was completed within 24 hours of admission, on the day shift, as this aligned with their schedule and usual contact with the patient. The independent raters used direct observation and information from the medical record to complete the CVONA. Throughout the study, those making CVONA ratings did not discuss their ratings with the nurses who rated the patient on the LoR and SRS. As mentioned above, all staff participating in this study were instructed that ratings made on the measures were not to be used to plan or modify clinical care. Consequently all ratings were maintained as confidential research data and not recorded in the medical record. Hence, those making CVONA ratings were blind to nurses' ratings on the LoR and SRS and vice-versa.

Subsequently, nurses rated the LoR and SRS on every participant twice per day, once on the day shift and again on the evening or night shift, depending on the patient's sleep/wake patterns. Ratings were spaced at least 8 hours apart on three separate days according to the following schedule: (1) within 2-3 days of admission, (2) within 5-6 days of admission, and (3) within 8-9 days of admission. Independent raters completed the CVONA for the same participants, once during daytime hours, adhering to the same schedule: (1) within 2-3 days of admission, (2) within 5-6 days of admission, and (3) within 8-9 days of admission.

\section{Data Analysis}

Primarily to assess item viability, preliminary CVONA item data obtained at each time point (admission, and 2-3 days, 5-6 days, and 8-9 days post-admission) were examined in 4 
separate Rasch analyses. In each of these analyses, items were eliminated from the pool until Infit and Outfit statistics for all items were within acceptable limits (.6 to 1.4).

Following these initial analyses, two potential CVONA measures were assembled. CVONA-I included only items that showed good fit to the underlying construct (i.e., Infit and Outfit $>.6$ and $<1.4$ ). The CVONA-II included CVONA-I items plus 5 additional items that demonstrated good fit on 2 or 3 of the 4 Rasch analyses.

Receiver operating characteristic (ROC), sensitivity and specificity of the CVONA-I and CVONA-II were then evaluated relative to independent assessment of need for visual observation (LoR) and supervision (SRS).

\section{Results}

\section{Rasch analyses by evaluation time points}

Separate Rasch analyses were conducted on data obtained at each of the four evaluation time points. The number of items meeting a priori fit criteria, i.e., both Infit and Outfit $>.6$ and $<1.40$ varied from 8 to 16 across the four time points. After eliminating misfitting items, Rasch metrics and Cronbach's alpha indicated acceptable internal consistency and construct validity for the measure at each time point although the number of items with acceptable fit varied across time points (see Table 1).

Insert Table 1 about here

\section{CVONA-I and CVONA-II}

CVONA-I was assembled from 8 items that met fit criteria at all evaluation time points. CVONA-II consisted of CVONA-I plus an additional 5 items that met fit criteria at 2 or 3 
Need for Constant Visual Observation

evaluation time points (see Table 2). Table 3 shows that internal consistency and construct validity for these measures was satisfactory at each evaluation time point.

Insert Tables $2 \& 3$ about here

\section{ROC analyses of CVONA-I and CVONA-II}

LoR and SRS served as concurrent measures for assessing the validity of CVONA-I and CVONA-II. A participant was considered to require $\mathrm{CVO}$ if one or both raters at a given time point rated the participant 3 or 4 on the LoR. A rating on the SRS of 10 or higher was considered as a secondary criterion for the need for constant visual observation.

Across the 4 evaluation time points, 2 ratings (daily and evening or night) were obtained at each time point on the LoR and on the SRS in $79-90 \%$ of the cases. In cases in which two ratings were available for the same participant at the same time point, the two raters agreed that the participant met the criterion for CVO on the LoR (score of 3 or 4 ) in $90-95 \%$ of cases. Raters agreed on the SRS criterion (score $\geq 10$ ) in $93-96 \%$ of the cases. Agreement between the two dichotomous concurrent measures ( $L O R \geq 3, S R S \geq 10$ ) ranged from $90-93 \%$ across the 4 evaluation time points.

Tables 5 and 6 show results of the ROC analyses of CVONA-I and CVONA-II at each time point for the two dichotomous concurrent measures (LoR, SRS). Both the CVONA-I and CVONA-II were significantly associated with thesemeasures, as indicated by area under the curve (AUC) statistics. (A chance relationship between the CVONA measures and criterion measures would result in an AUC of .50) Sensitivity and specificity of the CVONA measures were examined. Because false negatives were of primary concern, i.e., indicating that someone 
is NOT a safety risk when in fact the risk is significant, a cut off score was determined that resulted in approximately $90 \%$ sensitivity. Sensitivity at this level indicates a concomitantly low false negative rate, i.e., $<10 \%$. Cutoff scores and associated sensitivity values are displayed in Tables 4 and 5 as is the false positive rate (1-specificity).

Insert Tables 4 \& 5 about here

\section{DISCUSSION}

Creating and maintaining a culture of safety for rehabilitation inpatients with TBI is a "formidable undertaking," and the daily considerations that must be given to patient safety are some of the most difficult and demanding in terms of staff numbers and time. ${ }^{23}$ Though many safety interventions may be utilized, minimizing the use of physical restraints is an overarching goal. As such, the use of CVO has become increasingly prevalent and possibly overutilized. Unfortunately, extensive use of CVO can impose significant cost to health care organizations due to increased staffing, the inconsistency in which least-restrictive methods are employed, and a lack of standardized protocols. This study aimed to develop a tool based on the ABS to better guide decision-making regarding initiation and weaning of CVO.

The CVONA is a measure that assesses cognitive/communication, behavioral/emotional, and physical activities that may jeopardize safety. Activities and behaviors that potentially put the patient at risk appear to represent the construct that unifies items on the linear Rasch dimension. Both the CVONA-I and CVONA-II show acceptable internal reliability and consistency when administered at various times during inpatient rehabilitation. Associations with other indicators of need for CVO provide evidence of concurrent validity. However, when 
compared to these other indicators, CVONA measures show a high false positive rate in the context of good sensitivity. Of course the criterion indicators (LoR and SRS) also are not perfectly reliable and valid. Rater agreement on these measures was high but less than 100\%. Furthermore, in current practice, there is no generally acceptable protocol to determine CVO. After transfer to inpatient rehabilitation, patients may remain on CVO (or not) primarily because they were on CVO before transfer and remain on CVO (or not) for prolonged periods of time based on this initial decision. Indicators like LoR and SRS reflect the patient's current status but do not necessarily reflect actual need for CVO. In addition, completion of the CVONA required increased attention to patient behaviors and may have identified patients who in fact needed CVO but were not currently receiving it, that is, some false positives may not have been false.

While psychometric properties of the shorter (CVONA-I) and longer (CVONA-II) versions of this measure are similar, the cutoff scores for the longer version are more stable over time (see Table 4). The additional items in this longer version most likely support this stability and its use is recommended over the shorter version, particularly in future research. Most of the items that survived initial Rasch analysis reflected the patients' cognitive status or behavioral self-control rather than physical/motor features like pulling at tubes or balance. It may be that these cognitive and behavioral features were more pervasive and hence more easily and reliably observed than physical behaviors which occur more intermittently. In any case, some of the items that were eliminated in this initial psychometric analysis but have been traditional red flags for high risk patients, e.g., pulling at tubes/restraints, wandering, poor/unpredictable balance, and inability to express needs, may also merit further evaluation in future studies.

\section{Limitations}


Need for Constant Visual Observation

A true gold standard is not available to evaluate a new metric like the CVONA. While the absence of a gold standard challenges accurate estimation of the sensitivity and specificity of a measure, this initial evaluation indicates a high false positive rate which may lead to unnecessary and costly application of CVO in some cases. Although the current versions of the CVONA fit a linear Rasch model, some items that did not fit the model may be significant indicators of the need for $\mathrm{CVO}$, as mentioned previously. Our sample size did not allow for definitive examination of the dimensionality of the CVONA and it is possible that a precise measure of CVONA requires a multi-dimensional scale or scales. Thus, despite a relatively substantial number of participants recruited across multiple institutions in this study, additional studies with large, representative samples are needed to further develop a measure like CVONA and confirm its validity and the generalizability of its use.

\section{Conclusions}

Our results indicate that the CVONA had adequate sensitivity for identification of behaviors requiring $\mathrm{CVO}$. The high false positive rate suggests that $\mathrm{CVO}$ may be inappropriately recommended by the CVONA for some patients who do not require such intervention (at a potential cost to the organization). However, in light of the unavailability of a true gold standard criterion measure and the possibility that some false positive are in fact true positives, this cost must be considered versus the benefit in risk mitigation associated with false negatives.

As these results suggest, the CVONA may be considered as a complementary objective metric to improve consistency in clinical judgment regarding the need for CVO, but should not be considered prescriptive. In other words, while the CVONA should not be considered as a substitute for good clinical judgment, the use of such an objective measure is expected to help 
Need for Constant Visual Observation

standardize the assessment of behaviors that may jeopardize safety. In this way, its use may improve the consistency of such assessments particularly across time and changes in staff who are responsible for making clinical decisions about the need for CVO. Although this preliminary evidence suggests that the CVONA may adequately identify need for CVO, further prospective studies are necessary to validate its use for the identification of at-risk patients. A more definitive test of the validity and usefulness of the CVONA will require additional prospective study to determine whether its use results in fewer adverse events (such as, falls, potentially harmful behaviors to self or others) as well as more prudent and cost-effect use of CVO than current practice.

\section{References}

1. Beaulieu C, Wertheimer J, Pickett L, Spierre L, Schnorbus T, Healy W, Jones, A. Behavioral management on an acute brain injury unit: Evaluating the effectiveness of an interdisciplinary training program. Journal of Head Trauma Rehabilitation 2008;23(5):96102.

2. Becker C. Nursing care of the brain injury patient on a locked neurobehavioral unit. Rehabilitaton Nursing 2012;37(4):171-5.

3. Lequerica AH, Rapport LJ, Loeher K, Axelrod BN, Vangel SJ Jr, Hanks RA. Agitation in acquired brain injury: impact on acute rehabilitation therapies. Journal of Head Trauma Rehabilitation. 2007;22(3);177-83.

4. Lombard L, Zafonte R. Agitation after traumatic brain injury: Considerations and treatment options. American Journal of Physical Medicine and Rehabilitation 2005;84(10):797-812.

5. Nott, MT, Chapparo C, Baguley IJ. Agitation following traumatic brain injury. An Australian sample. Brain Injury 2006;20(11):1175-82.

6. Nott MT, Chapparo C, Heard R, Baguley IJ. Patterns of agitated behavior during acute brain injury rehabilitation. Brain Injury 2010;24(10):1214-21.

7. Scherder EJ, Bogen T, Eggermont LH, Hamers JP, Swaab DF. The more physical activity, the more agitation in dementia. Interntional Psychogeriatrics 2010;22(8):1203-8. 
8. Engberg J, Castle N, McCaffrey D. Physical restraint initiation in nursing homes and subsequent resident health. Gerontologist 2008;48(4):442-52.

9. Amato S, Salter JP, Mion LC. Physical restraint reduction in the acute rehabilitation setting: a quality improvement study. Rehabilitation Nursing 2006;31(6):235-41.

10. Votruba K, Rapport L, Vangel S, Hanks R, Lequerica A, Whitman R, Langenecker S. Impulsivity and traumatic brain injury: The relations among behavioral observation, performance, measures, and rating scales. Journal of Head Trauma Rehabiliattion 2008;23(2):65-73.

11. Bogner JA, Corrigan JD, Fugate L, Mysiw WJ, Clinchot D. Role of agitation in prediction of outcomes after traumatic brain injury. American Journal of Physical Medicine and Rehabilitation 2001;80(9):636-44.

12. Amato S, Resan M, Mion L. The feasibility, reliability, and clinical utility of the Agitated Behavior Scale in brain-injured rehabilitation patients. Rehabilitation Nursing 2012;37(1):1924.

13. Wolffbrandt MM, Poulsen I, Engberg AW, Hornnes N. Occurrence and severity of agitated behavior after severe traumatic brain injury. Rehabilitation Nursing 2013;38(3):133-41.

14. Rosario ER, Kaplan SE, Khonsari S, Patterson D. Predicting and assessing fall risk in an acute inpatient rehabilitation facility. Rehabilitation Nursing 2014;3:86-93.

15. Boswell D, Ramsey J, Smith M, Wagers B. (2001). The cost-effectiveness of a patient-sitter program in an acute care hospital: A test of impact of sitters on the incidence of falls and patient satisfaction. Quality Management in Health Care 2001;10(1):10-6.

16. Harding AD. Observation assistants: sitter effectiveness and industry measures. Nursing Economics 2010;28(5):330-6.

17. Laws D, Crawford CL. Alternative strategies to constant patient observation and sitters. Journal of Nursing Administration 2013;43(10):497-501.

18. Eastwood E, Schechtman J. Direct observation nursing: Adverse patient behaviors and functional outcomes. Nursing Economics 1999;17(2):96-102.

19. Riedel R, Shaw V. Nursing management of patients with brain injury requiring one-on-one care. Rehabilitation Nursing. 1997;22(1):36-9.

20. Joint Commission. National patient safety goals effective January 1, 2015. Hospital accreditation program. Oakbrook Terrace, IL; 2012. http://www.jointcommission.org/standards information/npsgs.aspx. 
21. Boake C. Supervision Rating Scale: A measure of functional outcome from brain injury. Archives of Physical Medicine and Rehabilitaton 1996;77:765-72.

22. Bogner J, Corrigan JD, Stange M, Rabold D. Reliability of the Agitated Behavior Scale. Journal of Head Trauma Rehabilitation 1999;14:91-6.

23. Pai AB, Zadov Y, Hickman A. Patient safety in rehabilitation medicine: Traumatic brain injury. Physical Medicine Rehabilitation Clinics of North America. 2012;23:349-370. 
Need for Constant Visual Observation

\begin{tabular}{|l|c|c|c|c|}
\hline \multicolumn{2}{|l|}{ Table 1. Rasch analyses by evaluation time points } \\
\hline \hline & Admission & Days 2-3 & Days 5-6 & Days 8-9 \\
\hline \hline $\begin{array}{l}\text { Number of items with } \\
\text { acceptable fit }\end{array}$ & 16 & 8 & 13 & 13 \\
\hline $\begin{array}{l}\text { Person } \\
\text { Separation/Reliability }\end{array}$ & $.86 / 2.53$ & $.86 / 2.48$ & $.87 / 2.60$ & $.88 / 2.72$ \\
\hline $\begin{array}{l}\text { Item } \\
\text { Separation/Reliability }\end{array}$ & $.99 / 8.14$ & $.99 / 10.64$ & $.99 / 8.55$ & $.99 / 8.32$ \\
\hline Cronbach's Alpha & .91 & .80 & .91 & .93 \\
\hline
\end{tabular}




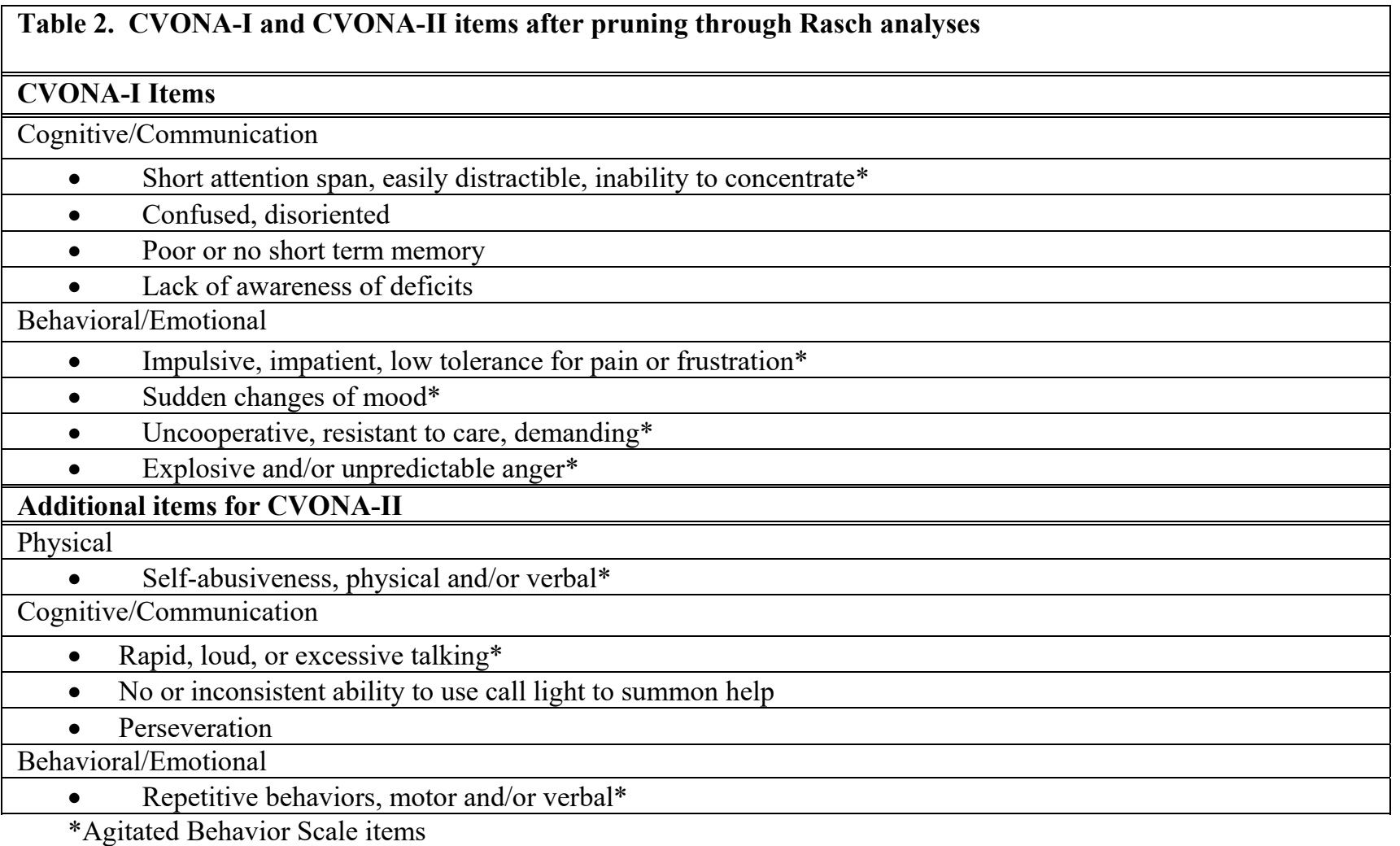


Need for Constant Visual Observation

\begin{tabular}{|c|c|c|c|c|c|c|c|c|}
\hline & \multicolumn{2}{|c|}{ Admission } & \multicolumn{2}{|c|}{ Days 2-3 } & \multicolumn{2}{|c|}{ Days 5-6 } & \multicolumn{2}{|c|}{ Days 8-9 } \\
\hline & CVONA-I & CVONA-II & CVONA-I & CVONA-II & CVONA-I & CVONA-II & $\begin{array}{l}\text { CVONA-I } \\
\end{array}$ & CVONA-II \\
\hline $\begin{array}{l}\text { Person } \\
\text { Separation/Reliability }\end{array}$ & $.85 / 2.43$ & $.87 / 2.54$ & $.86 / 2.48$ & $.85 / 2.37$ & $.86 / 2.44$ & $.87 / 2.56$ & $.88 / 2.77$ & $.88 / 2.65$ \\
\hline $\begin{array}{l}\text { Item } \\
\text { Separation/Reliability }\end{array}$ & $.99 / 10.30$ & .99/8.67 & $\begin{array}{c}.99 / 10.6 \\
3 \\
\end{array}$ & $.98 / 7.50$ & $.99 / 9.78$ & $.99 / 8.31$ & $.99 / 9.80$ & $.99 / 8.48$ \\
\hline Cronbach's Alpha & .87 & .90 & .88 & 90 & 89 & .92 & .91 & .93 \\
\hline
\end{tabular}


Need for Constant Visual Observation

\begin{tabular}{|c|c|c|c|c|c|c|c|c|}
\hline & \multicolumn{2}{|c|}{$\begin{array}{l}\text { Admission } \\
(\mathrm{n}=133)\end{array}$} & \multicolumn{2}{|c|}{$\begin{array}{c}\text { Days 2-3 } \\
(\mathrm{n}=134)\end{array}$} & \multicolumn{2}{|c|}{$\begin{array}{c}\text { Days 5-6 } \\
(\mathrm{n}=134)\end{array}$} & \multicolumn{2}{|c|}{$\begin{array}{c}\text { Days 8-9 } \\
(\mathrm{n}=126)\end{array}$} \\
\hline & CVONA-I & CVONA-II & CVONA-I & CVONA-II & CVONA-I & CVONA-II & CVONA-I & CVONA-II \\
\hline $\begin{array}{l}\text { Area Under the Curve } \\
\text { (AUC) }\end{array}$ & .832 & .831 & .765 & .766 & .769 & .768 & .824 & .838 \\
\hline $\mathrm{P}$ & $<.001$ & $<.001$ & .047 & .047 & .041 & .042 & .037 & .037 \\
\hline CVO cutoff & $\geq 12$ & $\geq 17$ & $\geq 11$ & $\geq 16$ & $\geq 10$ & $\geq 16$ & $\geq 9$ & $\geq 16$ \\
\hline Sensitivity & $89 \%$ & $89 \%$ & $93 \%$ & $93 \%$ & $90 \%$ & $91 \%$ & $92 \%$ & $93 \%$ \\
\hline $\begin{array}{l}\text { False positive rate } \\
\text { (1-specificity) }\end{array}$ & $29 \%$ & $33 \%$ & $48 \%$ & $46 \%$ & $50 \%$ & $55 \%$ & $56 \%$ & $46 \%$ \\
\hline
\end{tabular}


Need for Constant Visual Observation

\begin{tabular}{|c|c|c|c|c|c|c|c|c|}
\hline & \multicolumn{2}{|c|}{$\begin{array}{l}\text { Admission } \\
(\mathrm{n}=133)\end{array}$} & \multicolumn{2}{|c|}{$\begin{array}{c}\text { Days 2-3 } \\
(n=134)\end{array}$} & \multicolumn{2}{|c|}{$\begin{array}{c}\text { Days 5-6 } \\
(\mathrm{n}=134) \\
\end{array}$} & \multicolumn{2}{|c|}{$\begin{array}{c}\text { Days 8-9 } \\
(n=126)\end{array}$} \\
\hline & CVONA-I & CVONA-II & CVONA-I & CVONA-II & CVONA-I & CVONA-II & CVONA-I & CVONA-II \\
\hline $\begin{array}{l}\text { Area Under the Curve } \\
\text { (AUC) }\end{array}$ & .805 & .803 & .746 & .714 & .769 & .776 & .799 & .813 \\
\hline $\mathrm{P}$ & $<.001$ & $<.001$ & .047 & .049 & .043 & .043 & .041 & .040 \\
\hline CVO cutoff & $\geq 11$ & $\geq 16$ & $\geq 11$ & $\geq 16$ & $\geq 10$ & $\geq 16$ & $\geq 9$ & $\geq 16$ \\
\hline Sensitivity & $92 \%$ & $90 \%$ & $92 \%$ & $92 \%$ & $89 \%$ & $91 \%$ & $91 \%$ & $89 \%$ \\
\hline $\begin{array}{l}\text { False positive rate } \\
\text { (1-specificity) }\end{array}$ & $45 \%$ & $45 \%$ & $52 \%$ & $50 \%$ & $51 \%$ & $53 \%$ & $55 \%$ & $47 \%$ \\
\hline
\end{tabular}




\section{Constant Visual Observation Needs Assessment (CVONA)}

Center Number:

Rater Initials:

Date:

Subject Number: Time:

Scoring:

$\mathbf{1}=$ absent: the behavior is not present

$\mathbf{2}$ = present to a slight degree: the behavior is present but does not prevent the conduct of other, contextually appropriate behavior. (The individual may redirect spontaneously, or the continuation of the agitated/unsafe behavior does not disrupt appropriate behavior.)

$\mathbf{3}$ = present to a moderate degree: the individual needs to be redirected from an agitated/unsafe behavior to an appropriate behavior, but benefits from such cueing.

4 = present to an extreme degree: the individual is not able to engage in appropriate/safe behavior even when external cueing or redirection is provided.

CIRCLE SCORE. DO NOT LEAVE BLANKS (Note: Bold = 14 ABS items)

\begin{tabular}{|c|c|c|c|c|}
\hline Physical & & & & \\
\hline Pulling at tubes, restraints, etc. & 1 & 2 & 3 & 4 \\
\hline Rocking, rubbing, moaning or other self stimulating behavior & 1 & 2 & 3 & 4 \\
\hline Self-abusiveness, physical and/or verbal & 1 & 2 & 3 & 4 \\
\hline Wandering from treatment areas & 1 & 2 & 3 & 4 \\
\hline Restlessness, pacing, excessive movement & 1 & 2 & 3 & 4 \\
\hline Poor/unpredictable balance & 1 & 2 & 3 & 4 \\
\hline History of falls $<30$ days. ( 1 if no known history / 4 if any falls within last 30 days) & 1 & 2 & 3 & 4 \\
\hline History of elopement & 1 & 2 & 3 & 4 \\
\hline Dizziness & 1 & 2 & 3 & 4 \\
\hline Incontinence & 1 & 2 & 3 & 4 \\
\hline Inability to toilet self & 1 & 2 & 3 & 4 \\
\hline \multicolumn{5}{|l|}{ Cognitive/Communication } \\
\hline Short attention span, easily distractible, inability to concentrate & 1 & 2 & 3 & 4 \\
\hline Rapid, loud, or excessive talking & 1 & 2 & 3 & 4 \\
\hline Confused, disoriented & 1 & 2 & 3 & 4 \\
\hline Delusional and/or hallucinating & 1 & 2 & 3 & 4 \\
\hline Poor or no short term memory & 1 & 2 & 3 & 4 \\
\hline Lack of awareness of deficits & 1 & 2 & 3 & 4 \\
\hline No or inconsistent ability to use call light to summon help & 1 & 2 & 3 & 4 \\
\hline Perseveration & 1 & 2 & 3 & 4 \\
\hline Confabulation & 1 & 2 & 3 & 4 \\
\hline Aphasia, inability to express needs & 1 & 2 & 3 & 4 \\
\hline \multicolumn{5}{|l|}{ Behavioral/Emotional } \\
\hline Impulsive, impatient, low tolerance for pain or frustration & 1 & 2 & 3 & 4 \\
\hline Sudden changes of mood & 1 & 2 & 3 & 4 \\
\hline Uncooperative, resistant to care, demanding & 1 & 2 & 3 & 4 \\
\hline Repetitive behaviors, motor and/or verbal & 1 & 2 & 3 & 4 \\
\hline Easily initiated or excessive crying and/or laughter & 1 & 2 & 3 & 4 \\
\hline Explosive and/or unpredictable anger & 1 & 2 & 3 & 4 \\
\hline Violent and/or threatening toward people or property & 1 & 2 & 3 & 4 \\
\hline History of aggression & 1 & 2 & 3 & 4 \\
\hline
\end{tabular}

Center Number:

Rater Initials:
Subject Number:

Date: Time: 


\section{SUPERVISION RATING SCALE (SRS)}

Independent

01=Alone, Independent

02=Unsupervised at night, sometimes during day

Overnight supervision

03=Supervised only at night

Part Time supervision

04=Supervised at night and selected day times.

05=Supervised at night and part-time during day; not supervised during working hours (full time)

06=Supervised at night and most of day except for few unsupervised hours.

07=Only unsupervised for periods less than one hour at a time.

\section{Full-time indirect supervision}

08=Full time indirect supervision; does not check more than once every $\mathbf{3 0}$ minutes

09=Same as 08, and requires overnight safety precautions (lock, etc.)

\section{Full time direct supervision}

$10=$ Full time direct supervision; checked more than once every thirty minutes

11=Full time direct supervision in confined, controlled setting.

12=Same as 11, but with constant visual watch

$13=$ Person is in physical restraints.

\section{LEVEL of RISK (LoR)}

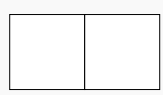

I -No known safety risk (no need for CVO, standard nursing staffing assignment

II -Slight safety risk (no need for CVO, use interventions such as bed and chair alarms, specialized beds, frequent room checks, Secure Care/Wanderguard system)

III -High safety risk (probable need for CVO, daily interdisciplinary team review of need for CVO, implement Level II safety measures, consider use of more restrictive measures including approved restraints

IV-Extreme safety risk (definite need for CVO; daily interdisciplinary team review of ongoing need for CVO, implement Level II safety measures, consider use of more restrictive measures including approved restraints 